\title{
NORME THÉORIQUE ET MISES EN PRATIQUE : le cas de Gratien du Pont $(1534,1539)$
}

\section{Véronique Montagne}

Université Nice Sophia Antipolis

Laboratoire CNRS/ BCL/ UMR 7320

\section{Résumé}

Les normes prosodiques modernes se mettent progressivement en place à la Renaissance, dans des traités de seconde rhétorique comme celui du toulousain Gratien du Pont, intitulé L'art et science de rhetoricque metriffiée (1539). Ce dernier ouvrage présente un cas où l'auteur construit une norme qui correspond parfaitement à son propre travail poétique, tel qu'il est représenté par les Controverses des sexes masculin et femenin parues en 1534. Cette norme est en revanche déjà désuète dans le contexte poétique des années 1540-1550, qu'il s'agisse de la théorisation ou de la pratique de cette dernière, à l'exception notable de la Requeste faicte et baillée par les dames de la ville de Tolose, qui a été rédigée dans les années 1540, avant d'être publié en 1555 .

\section{Mots-clés}

poésie, rhétorique, vernaculaire, normes, e muet, hiatus
Abstract

Modern prosodic standards set up during the French Renaissance, in second rhetoric treatises, like L'art et science de rhetoricque metriffiée (1539) written by Gratien du Pont, from Toulouse. This treatise present a poetic standard which match perfectly with his own poetic work, represented by the Controverses des sexes masculin et femenin (1534). This standard is however yet outdated in the poetic context of the years 1540-1550, whether theoretical, whether practical, except the anonymous Requeste faicte et baillée par les dames de la ville de Tolose, written during the 1540' and published in 1555 .

\section{Keywords}

poetry, rhetoric, vernacular, norms, silent e, hiatus 
Le toulousain Gratien du Pont est l'auteur d'une rhétorique seconde, publiée en 1539, qui s'intitule l'Art et science de rhetoricque metriffiée. Il s'agit d'un texte normatif - probablement le dernier exemple de ce genre (Patterson 1935, p. 3 ou Monferran 2011) -, qui traite des formes de la poésie et qui légifère sur la métrique et la versification en français. Le titre du texte de Gratien du Pont évoque L'art de rhétorique vulgaire de Jean Molinet, paru en 1493, mais il évoque aussi et surtout le Collège des Art et Science de Rhétorique, nom porté par les organisateurs des Jeux floraux toulousains au Xvi ${ }^{\mathrm{e}}$ siècle - un concours poétique annuel -, dont Gratien du Pont a fait partie dans les années 1530-1540. Cet ouvrage est à lire en relation avec les autres rhétoriques « métriffiées », parues entre 1490 et 1540, comme L'art de rhétorique vulgaire (1493) de Jean Molinet (voir Monferran 2015), comme l'Instructif de seconde rhétorique (circa 1500) qui ouvre le Jardin de plaisance et fleur de rhétorique, comme Le Grand et Vrai Art de pleine rhétorique (1521) de Pierre Fabri ou encore comme L'art et science de rhétorique vulgaire (1524-1525), qui est un ouvrage anonyme. Il est également à mettre en relation avec la poésie qui a cours depuis la fin du Xv $\mathrm{Xv}^{\mathrm{e}}$ siècle et jusqu'aux années 1530-1540 : en tout premier lieu, le recueil du même Gratien du Pont, intitulé Controverses des sexes masculin et femenin, paru en 1534 ; mais aussi les textes produits pour les Jeux Floraux à partir de 1539 (De Gélis et Anglade 1933) ou des œuvres de Jean Meschinot, de Jean Lemaire de Belges et de Clément Marot. À ce paradigme poétique, on ajoutera un texte rédigé dans les années 1540, mais seulement publié en 1555, La requeste faicte et baillée par les dames de la ville de Tolose.

Le rapprochement de ces deux types de corpus - théorique et pratique - montre que coexistent alors des normes et des usages poétiques et prosodiques différents dans un " même » espace géographique et social. Le texte de Gratien du Pont constitue un exemple de tentative d'instaurer une norme, dont nous verrons qu'elle ne rencontre pas le succès escompté, probablement parce qu'elle est parue dans un contexte linguistique inadapté, c'est-à-dire trop tard, ou trop tôt...

\section{UN TEXTE NORMATIF / LINGUISTIQUE DE LA NORME}

\subsection{Formes définitionnelles}

L'Art et science de rhétoricque metriffiée est un ouvrage composé de règles, lesquelles sont présentées sous la forme de définitions conventionnelles, avec un lexique métalinguistique, dans des énoncés de type stipulatoire. La perspective peut être onomasiologique, comme en témoigne l'usage récurrent du verbe «s'appeler» :

Rithme ruralle est celle qui ne Rithme en fin de ligne que d'une lettre, et s'appelle « rurale » pource que les ruraulx et rusticques en usent. (Du Pont 2012, p. 124) 
Dans cette perspective, la définition peut alors prendre la forme d'un énoncé polyphonique, avec la présence d'un discours rapporté, introduit par la forme passive « est $\operatorname{dit}(\mathrm{e}) »$ :

Et notez que proprement rithme «platte » est dicte, quant deux lignes consequtives, Masculines et femenines se trouvent d'ung mesme son et terminaison... (Du Pont 2012, p. 123)

On aura noté la perspective orthonymique dans cette dernière définition, avec l'utilisation de l'adverbe " proprement », qui souligne que l'usage qui est fait de la notion est le seul valide.

La définition peut être de type sémasiologique, comme en témoigne l'usage de la phrase attributive :

Synalephe declairé en langue Françoyse, n'est aultre chose que abbreviation d'ungne syllabe entre deux voyelles, mengeant l'unne d'ycelles. (Du Pont 2012, p. 234)

On signalera ici le dialogisme de la négation, qui suppose une assertion différente, une autre norme, sur laquelle nous reviendrons plus loin.

\subsection{Un acte illocutoire assertif et commissif}

L'utilisation de définitions dans des phrases assertives, le recours à l'orthonymie, qui signale la primauté d'un sens propre, originel, auquel l'auteur entend se conformer, la présence de discours rapportés qui souligne elle aussi une conformité à l'usage dominant caractérisent ainsi un propos qui relève d'un acte illocutoire à la fois assertif et commissif.

Ainsi, la perspective onomasiologique et orthonymique s'accompagne-t-elle parfois d'une discussion sur la pertinence de telle ou telle autre dénomination, à l'occasion de laquelle l'auteur prend résolument parti :

Les Rhetoriciens, et Autheurs modernes, nomment une espece de Rithme " Arbre fourchu », laquelle ledict Fabri nomme " Lay », comme avez ouy. Mais selon nostre advys, ledict tiltre «Arbre fourchu » est mieulx approprié à ladicte espece que «Lay», qui à ce propos ne veult rien dire. (Du Pont 2012, p. 169)

La pratique est très régulièrement injonctive et les recommandations passent par l'utilisation de verbes déontiques omniprésents, comme se faire (qui impose ici une lecture injonctive), devoir ou falloir :

Notez que lesdictz rondelletz ou triolletz se font à une syllabe ou plus jusques audict nombre. (ibid., p. 148) ${ }^{1}$

Et puys doibt rentrer et reprendre les deux premieres lignes du premier couplet, ou bien le premier mot, et aulcunesfoys le premier et second. (ibid., p. 151)

La cinquiesme ligne fault que soit de la mesure, forme et resonance, desdictes deux premieres. (ibid., p. 156)

1 Souligné par nous, comme dans les exemples qui suivent. 
Le lexique utilisé par l'auteur signale clairement l'entreprise normative. On note ainsi soixante-quatorze occurrences du mot règle dans le texte, par exemple lorsqu'il s'agit d'évoquer la règle des Anciens (ibid., p. 236) et la règle des Modernes (ibid., p. 249) en matière de synalèphe. Le terme est mis en relation parasynonymique avec le substantif compas, au début du texte :

Premierement nous equipparerons Rithme à musicque, car tout ainsi que Musicque requiert estre gouvernée par mesure et compas, par mainctes regles generalles, mainctes exceptions, differences, et façons de faire. Semblablement ladicte rithme en veult estre regie, gouvernée, et manifestée. (ibid., p. 96)

Le mot renvoie directement à la page de titre de l'Art et science de rhetoricque metriffiée, sur laquelle un personnage masculin tient un livre (voir page suivante). Ce personnage a la langue percée par le compas que manipule une main sortant de nuages. L'illustration est située dans un double cercle, dans lequel apparaît la devise loqui ad mensuram optimum, que l'on pourrait traduire par « le mieux est de parler selon la mesure », le terme "mesure » ayant ici le sens technique de "quantité métrique $»^{2}$. L'homme évoque Hercule, dont la langue n'est pas attachée à une chaîne, comme dans la fable rapportée par Lucien, mais à un compas : l'éloquence est ici mesurée de manière mathématique et la gravure annonce ainsi le projet normatif de Gratien du Pont. Hercule ne s'exprimera que d'une manière mesurée, évaluée ; il n'est pas celui qui enchaîne les hommes, mais celui dont la parole est réglée par un maître quasi-divin, dont la main descend du ciel pour donner ses injonctions métriques. Cette entreprise normative suppose une conception de la " règle ", qui est précisée par des éléments de métathéorie épars dans le texte. La première des deux principales caractéristiques de la « règle » est que celle-ci correspond à un usage, comme le souligne régulièrement le Toulousain : " le commun usaige vulgairement vous demonstre qu'on le doibt synalepher » (Du Pont 2012, p. 243). L'usage est défini comme correspondant à la " commune prolation » (ibid., p. 255), mais il est précisé que cette prolation est celle que les doctes conçoivent comme acceptable, et seulement eux. Le jugement de l'oreille - de l'oreille docte, donc - valide ou invalide un choix prosodique :

Notez que tousjours où il y a Regle, on la doibt observer si totallement, Nostre juge, qu'est l'oreille n'y contredict, nous entendons de l'oreille docte, non suspecte... (ibid., p. 243)

Lorsqu'il arrive que l'usage commun entre en contradiction avec les préférences de l'oreille docte, c'est cette dernière qui prévaut, parce qu'elle incarne, selon Gratien du Pont, la « raison » qui est à l'origine de la formulation d'une règle.

2 Le thème du compas n'est pas nouveau : avant d'être la marque de Christophe Plantin, à partir des années 1560, elle était ponctuellement celle d'Hémon Le Fèvre, libraire à Paris de 1509 à 1525, avec la devise teneo mensura (m) (Tervarent 2000, p. 140). 
Ainsi, à propos d'une absence régulière de synalèphe, que Gratien du Pont tient lui pour appropriée, il est précisé, à propos des contrevenants, « tel long usaige leur a adveuglé Dame rayson », avant la conclusion « pour le meilleur, on doibt favoriser et obeyr à la Regle, non croyre à soy-mesmes » (ibid., p. 244).

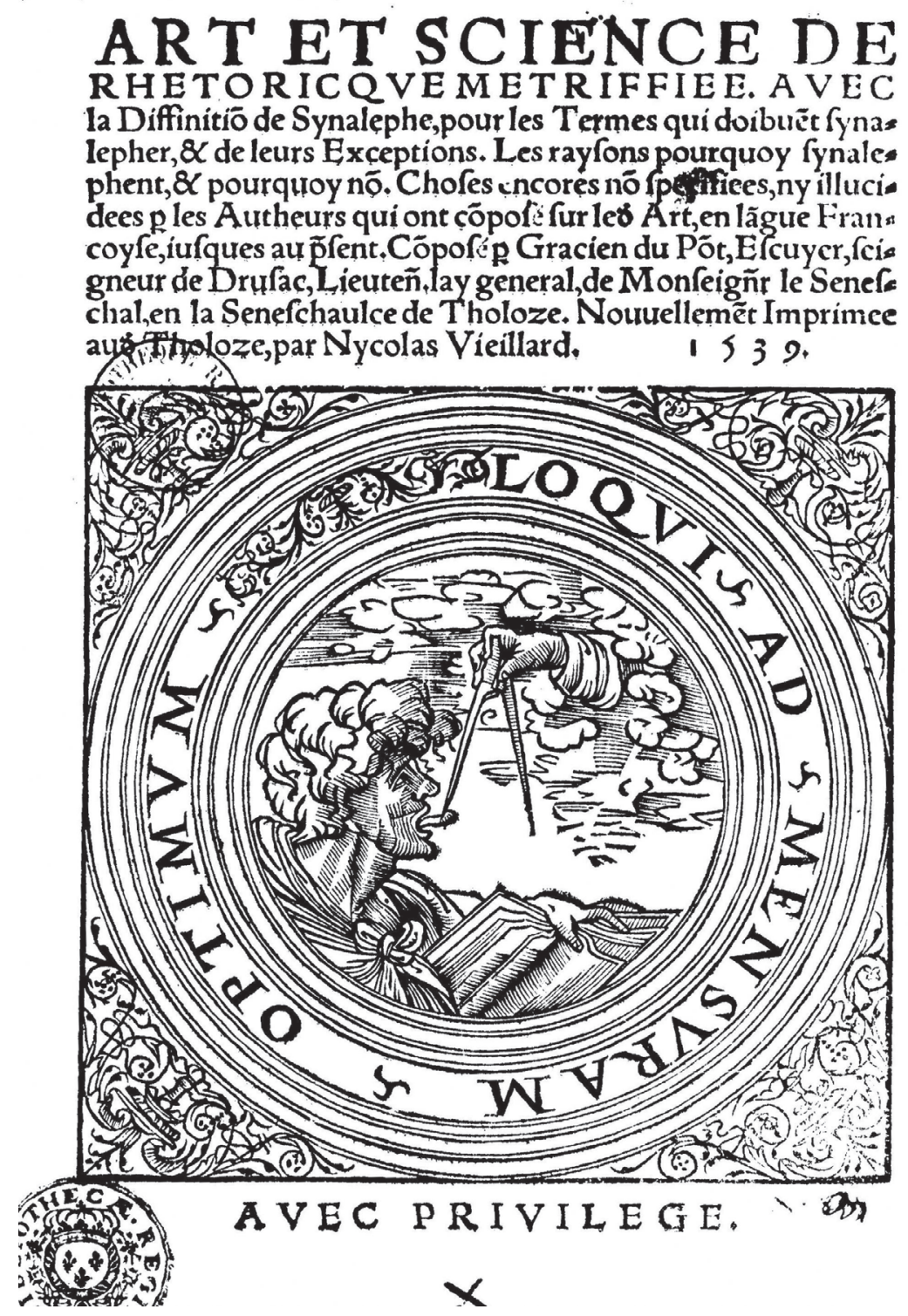

Page de titre de l'Art et science de rhetoricque metriffiée, Toulouse, Vieillard, 1539. 
Qui sont ces doctes qui sont seuls capables d'entendre raison et de proposer des règles considérées comme valides ? Il s'agit, en l'occurrence, de ceux que l'auteur appelle les «Anciens », en profitant, parfois, de leur silence sur tel ou tel autre point, pour imposer sa norme personnelle :

Lesdictz Anciens practiquoyent les motz et termes, tant masculins que femenins, que masculins feminisez, finissantz par voyelle masculine feminine, ou feminisée, en commenceant aultre mot, et terme par quelque voyelle que ce fust, estre synalephez, ne faisant mention des Exceptions qu'ilz practiquoyent, sans les divulguer en leurs Rhetoricques. Desquelles cy dessoubz nous ferons mention, et Exemples au moins d'ungne partie. (ibid., p. 255)

Toute règle entérinée par l'usage et la raison des doctes est supposée pérenne et l'existence d'une évolution, évoquée dans l'Art et science pour être contestée, est ainsi apparentée à une hérésie, dans un amalgame politique, religieux et culturel :

Suyvons le grand chemyn, que nostre mere saincte Esglyse nous a enseigné, et enseigne. Et laissons toutes sentes nouvelles indirectes, lesquelles conduysent ceulx qui les ensuyvent, au Gouffre de tout malheur, qu'est en l'abysme d'enfer. [...] Au temps qui court par certains de telz nouveaulx controuveurs, n'est chose ancienne qu'ilz ne veullent refformer. [...] Ilz refforment L'orthographie, les Regles termes, et mesures, tant en latin qu'en Françoys. Ce qu'ilz disent et font pour mal qu'il soyt, ilz disent que ainsy se doibt faire, et se faict au present. Et que il est receu. Combien que n'en ayons veu oncques Registre de reception (ny Arrest) que d'eulx mesmes qui sont juges et parties. (ibid., p. 238-239)

La deuxième caractéristique de la règle est qu'il s'agit d'un principe applicable et qui doit donc être appliqué : « tousjours où il y a Regle, on la doibt observer [...] totalement » (ibid., p. 243) précise ainsi le Toulousain. Cela l'autorise à dénoncer ce qu'il considère comme une incohérence du normand Pierre Fabri :

[...] la pluspart de ses exemples sont contraires à la loy et regles qu'il a baillées, comme verrez par son exemple cy dessoubz, lequel est faulx contre tout bon stille, proportion et mesure, car il vient contre sa mesme regle posée en l'huyctiesme fueillet de sadicte Rhetoricque... (ibid., p. 166 ; voir Fabri 1969, p. 14)

En gardant à l'esprit cette définition de la règle, je vais maintenant m'intéresser à la question de la prononciation du « e », qui concentre nombre de remarques directives dans le texte. Il s'agira de se demander si les recommandations de Gratien du Pont correspondent, d'une part, à celles des œuvres théoriques contemporaines et, d'autre part, à la pratique poétique également contemporaine.

\section{ADÉQUATION / INADÉQUATION}

\subsection{Théories grammaticales / phonétiques / prosodiques}

La question de la prononciation du « e » final concerne ce que Gratien du Pont comme bien d'autres (voir Du Pont 2012, p. 32-33) - appelle les mots féminins, 
comme « Eve, Femme, Dame, Donne, Habandonne, Belle, Mortelle, Pensée » ou « Diffamée ». Pour le Toulousain, le « e » final se prononce « o », qu'il soit placé derrière une consonne ou derrière une voyelle (ibid., p. 118). Or cette déclaration entre en contradiction avec l'évolution prosodique standard, qui est celle du Nord de la France, laquelle a amené un amuïssement presque total du « e » dit atone avant le début du XvI ${ }^{\mathrm{e}}$ siècle (Elwert 1965, p. 51) et qui sera total dans le langage quotidien à partir du premier tiers du XVII ${ }^{e}$ siècle (ibid., p. 43). Gratien du Pont, qui est natif du sud-ouest et où n'a pas lieu cette évolution prosodique, est en désaccord avec Pierre Fabri, qui parle de « demye syllabe » pour la dernière syllabe du vers comportant un «e » féminin (Fabri 1969, p. 5).

Dans L'éclaircissement de la langue française, paru en 1530, le grammairien John Palsgrave signale lui aussi que le «e » final se prononce «o » (Palsgrave 2003, p. 369). Les positions de Gratien du Pont et de John Palsgrave, en concurrence avec ce que signalent d'autres auteurs, montrent que différents usages cohabitent alors et que la disparition de ce « $\mathrm{e}$ » en position finale n'est pas encore une évidence ou ne l'est pas pour tout le monde.

Pour Gratien du Pont, dans les vers longs, lorsqu'un «e » est la dernière voyelle du vers, celui-ci se prononce plus ou moins distinctement, sans être compté métriquement. Gratien du Pont identifie ainsi des décasyllabes de dix ou de onze syllabes (Du Pont 2012, p. 108). Par ailleurs, pour le Toulousain, les deux parties du vers doivent être considérées de la même façon : le statut du «e » en fin de vers, prononcé mais non compté métriquement, doit donc être le même à la coupe, selon le principe qui veut qu'une règle doit être appliquée systématiquement. Le « $\mathrm{e}$ » à la coupe/ césure est donc prononcé, mais non compté : c'est le principe de la césure épique (voir ibid., p. 38-40).

En 1521, Pierre Fabri considérait lui aussi que ce «e » se prononçait (Mantovani 2000, p. 44), mais comme cela entraînait manifestement des difficultés de lecture eu égard à l'évolution de la prononciation de ce «e » en prose, il recommandait aux poètes de s'abstenir de la coupe féminine dans la mesure du possible (Fabri 1969, p. 97) et, dans le cas où cela n'était vraiment pas possible, c'est aussi la césure épique qui était recommandée (ibid., p. 98).

Or cette position est bien loin de faire l'unanimité et elle s'oppose ainsi à ce que recommande déjà Jean Molinet, dans L'art et science de rhétorique vulgaire (Monferran éd. 2015, p. 220-221), comme l'auteur anonyme de l'Instructif de seconde rhétorique ou encore l'auteur de l'Art et science de rhétorique vulgaire (Langlois éd. 1902, p. 268). Ces auteurs - qui publient leurs textes entre 1493 et 1525 - préconisent ainsi l'élision du «e » final d'un mot devant une voyelle lorsque le «e » est à la coupe et il est alors bien précisé que ce « $\mathrm{e}$ » ne se prononce pas (Langlois éd. 1902, p. 268-269 et introduction p. LXXXV). Ils rejettent donc 
le principe de la césure épique et celui de la prononciation du «e » féminin, choix qui sera entériné dans les années 1550 , notamment avec l'Art poétique français de Thomas Sébillet (1548).

Le parti pris d'une coupe nettement marquée est ressenti comme un particularisme toulousain par Jean Dupré, dans Le Palais des nobles dames (ca 1535). Au sujet de la coupe féminine, le poète précise ainsi :

[...] François plein de doctrine

L'ont en haut prix; Tholose la défend,

Disant raison que synalèphe offend

Le son du vers, vu qu'il faut qu'on repose

Sur le milieu... (Dupré 1534, n.p.)

\subsection{Pratiques poétiques}

La norme que propose Gratien du Pont au sujet du « e » et de la coupe féminine correspond-elle toutefois à un usage poétique et/ou devient-elle un usage poétique, indépendamment de ces recommandations théoriques qui vont globalement dans un sens opposé ? Pour répondre à cette question, j'évoquerai tout d'abord la poésie toulousaine, puis la poésie « française », cette partition - a priori paradoxale supposant un clivage déjà évoqué, sur lequel je reviendrai un peu plus loin.

\subsubsection{Poésie toulousaine: le cas des Controverses des sexes masculin et femenin}

Les principes exposés dans l'Art et science au sujet de la prononciation du « e » sont déjà mis en pratique dans les Controverses des sexes masculin et femenin (1534) où Gratien du Pont utilise régulièrement la césure épique. Il place souvent un « e » à la césure, qui doit être prononcé (dixit l'Art et Science), mais non comptabilisé dans le vers, bien qu'il soit placé devant une consonne (Du Pont 1534, $\mathrm{f}^{\circ}$ liii $\mathrm{v}^{\circ}$, liii $\mathrm{v}^{\circ}, \mathrm{xlv} \mathrm{v}^{\circ}$ ). Gratien du Pont utilise par ailleurs des finales en hiatus -ée, dans lesquelles le « e » final se prononce clairement ${ }^{3}$. La relation autodialogique de la théorie à la pratique est ici manifeste : en 1539, l'auteur justifie et explicite sa pratique poétique de 1534 par l'élaboration et l'explicitation de cette norme dans l'Art et science de rhetoricque vulgaire.

Les illustrations mettent en évidence la relation entre les deux textes. La page de titre de l'Art et science rappelle celle qui ouvre les Controverses des sexes masculin et femenin, du même auteur, texte paru cinq ans plus tôt (voir page 141 et page suivante). Le volume de l'Art et science est paru en 1539, chez Nicolas Vieillard, et celui des Controverses en 1534, chez Jacques Colomiès : l'illustration dont il est ici question n'est donc pas la marque des imprimeurs, mais le résultat d'un choix de l'auteur.

3 Dans les Controverses des sexes masculin et femenin, un terme comme privées compte ainsi pour trois syllabes (ibid., $f^{\circ}$ xiiii $r^{\circ}$ ). De nombreux exemples attestent que l'hiatus « -ée » est préservé (voir ibid., f xiiii $\mathrm{r}^{\circ}, \mathrm{f}^{\circ} \mathrm{xiiii} \mathrm{r}^{\circ}, \mathrm{f}^{\circ} \mathrm{xxvii} \mathrm{v}^{\circ}, \mathrm{f}^{\circ} \mathrm{xxx} \mathrm{r}^{\circ}, \mathrm{f}^{\circ} \mathrm{xlii} \mathrm{v}^{\circ}$ ou f $\mathrm{f}^{\circ} \mathrm{lvii} \mathrm{v}^{\circ}$ ). 


\subsubsection{Poésie toulousaine : le cas des poèmes récompensés aux Jeux Floraux}

Le texte de l'Art et science de rhetoricque metriffiée et la pratique proposée dans les Controverses ont-ils exercé une influence importante, notamment dans le milieu toulousain, sur les compositions proposées aux Jeux Floraux ? Les Actes et délibérations du collège de rhétorique (1513-1641), qui recensent les pièces poétiques couronnées entre 1539 et 1545 - période durant laquelle Gratien du Pont faisait partie du Collège de rhétorique - permettent de constater qu'au moment de la parution de l'Art et Science, la césure épique ne semble être jamais pratiquée et que l'élision à la césure entre un mot finissant par un «e » et un mot commençant par une autre voyelle est souvent possible (De Gélis et Anglade 1933, p. 27-28).

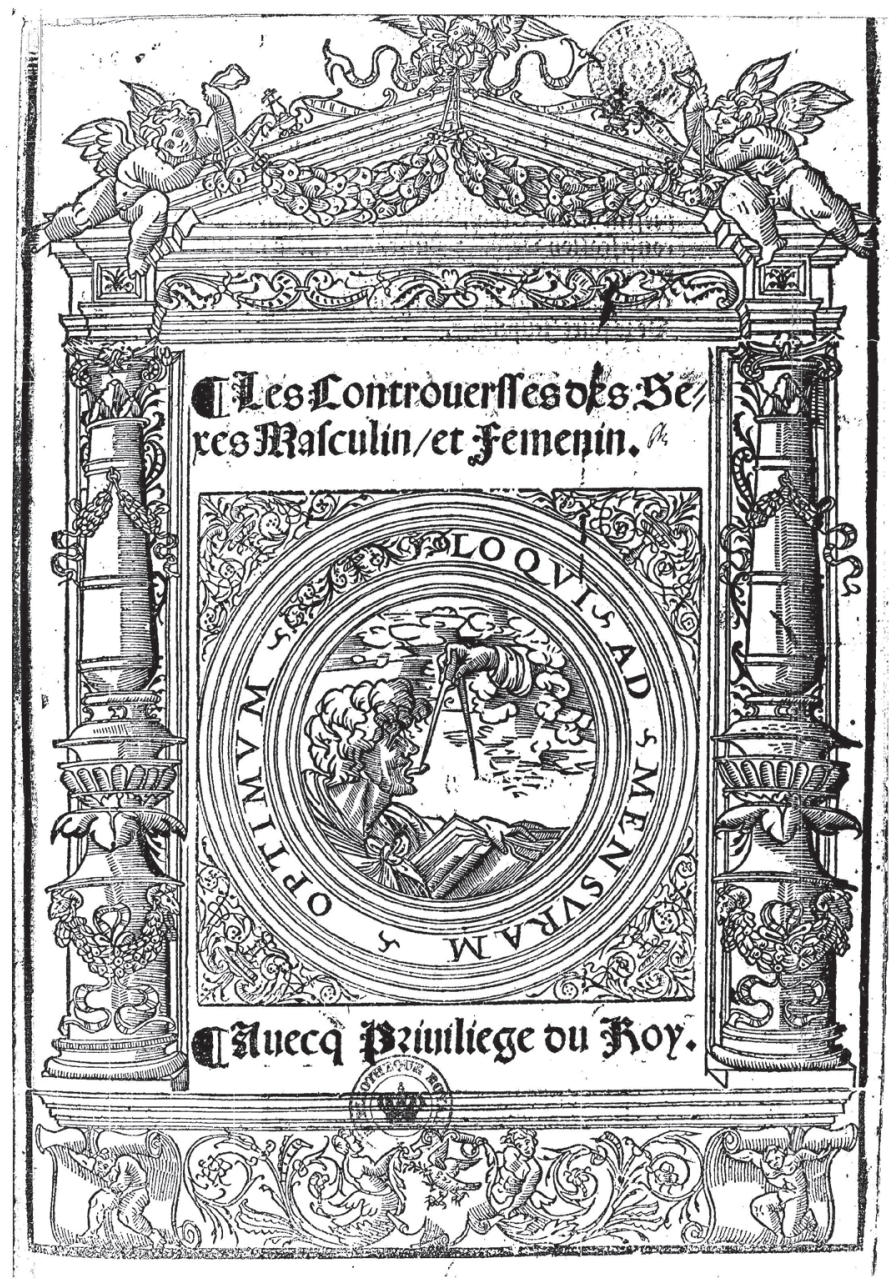


2.2.3. Poésie toulousaine : le cas de la Requeste faicte et baillée par les dames de la ville de Tolose

L'influence est toutefois plus manifeste sur la Requeste faicte et baillée par les dames de la ville de Tolose, qui a été rédigée dans les années 1540, avant d'être publié en 1555. Ce texte en deux parties - l'une en français, l'autre en occitan est un ouvrage anonyme (voir De Gélis 1912, p. 281 et 290). D'un point de vue thématique, la Requeste s'inspire du contenu misogyne des Controverses des sexes masculin et femenin (Courouau et Gardy 2003, p. 64 sqq.). Contrairement aux pièces récompensées aux Jeux Floraux à partir des années 1539, le texte illustre par ailleurs les compositions utilisées dans les Controverses (ibid., p. 66) et décrites dans l'Art et science et se signale par l'emploi omniprésent de la césure épique (ibid., p. 67 sqq.).

Pour comprendre les raisons de ces choix formels, il faut revenir aux Controverses et à une injonction que Gratien du Pont y adresse d'emblée à ses éventuelles contestataires :

Aussy Seigneurs : je vous prie humblement

Que sy respondre : voulez à l'argument.

Que je vous fais : de sexe masculin.

Sur les abuz: du sexe femenin.

Que vous sera : chose tresque facille

Me respondez: en Rythme et tel stille

Comme je argue : poinct pour poinct... (Du Pont $1534, \mathrm{f}^{\circ}$ ix $\mathrm{r}^{\circ}$ et $\mathrm{v}^{\circ}$ )

L'auteur annonce ainsi qu'il n'acceptera d'autre contestation, qu'il ne l'entendra, que si celle-ci est rédigée dans une langue conforme à la sienne, c'est-à-dire en français et en vers :

Pource de vous : aulcun ne se dispose.

De me respondre : en grec / latin / ne prose

En aultre langue : ne forme que je fais

Equiparables : soyent voz dictz à mes faictz... (ibid., $\mathrm{f}^{\circ}$ ix $\mathrm{r}^{\circ}$ et $\mathrm{v}^{\circ}$ )

On peut donc supposer que les choix formels des auteurs de la Requeste - qui différent de ceux de leurs compatriotes pour la même époque - relèvent d'un parti-pris énonciatif : il s'agit là de répondre à l'auteur misogyne incriminé, en utilisant sa langue (dans la première partie du texte) et/ou ses recommandations métriques (dans l'ensemble du texte), dans une œuvre qui serait donc caractérisée par un dialogisme interlocutif responsif.

\subsubsection{Poésie "française » (langue d'oül)}

L'écho à cette norme est-il manifeste chez les auteurs de langue d'oïl ? Dans l'Art et science (Du Pont 2012, p. 292), Gratien du Pont encense quelques auteurs, auxquels il reconnaît le mérite de ne pas avoir pratiqué l'élision systématique du «e » à la césure, comme Jean Meschinot (« autheur de la Lunette des Princes », 1493), 
Guillaume de Lorris et Jean de Meung (auteurs du Roman de la rose, 1402), Jacques Milet (auteur de La destruction de Troye, 1484), l'auteur anonyme de la rhétorique du Jardin de plaisance (ca 1501 ; voir Monferran 2015, p. 15 sqq.), Martin Le Franc (auteur du Champion des dames, ca 1489-1492), Guillaume Alexis (auteur du Blason de faulces Amours, fin $\mathrm{Xv}^{\mathrm{e}}$ siècle), Alain Chartier (auteur de La belle dame sans mercy, 1424), Octovien de Saint-Gelais (auteur du Séjour d'honneur, 1494), Blaise d'Auriol (auteur de La départie d'amour, 1508 ; voir Dawson 1921, p. 27), Guillaume de la Perrière et quelques autres auteurs toulousains ${ }^{4}$. De tous ces auteurs, il déclare qu'ils n'ont jamais recouru à l'élision du « e » à la césure.

Qu'en est-il de la pratique réelle des auteurs encensés ici ? Dans certains des textes cités, la question de la coupe, en l'occurrence féminine, ne se pose pas, puisque les mètres utilisés sont de longueur inférieure au décasyllabe. C'est le cas du Champion des dames de Martin Le Franc, du Blason des fausses amours de Guillaume Alexis, du Roman de la rose ou encore de La belle dame sans merci ou du Jardin de plaisance. Lorsque la question se pose, les auteurs recourent modérément à la coupe épique ${ }^{5}$ et n'évitent pas l'élision du « e » à la coupe : tel est par exemple le cas dans Les lunettes des princes de Jean Meschinot ${ }^{6}$ ou Le séjour d'honneur d'Octovien de Saint-Gelais'.

Donc, soit Gratien du Pont se concilie des autorités qui n'en sont pas vraiment, soit il veut soutenir que ces auteurs - dans les cas où la question se pose - ne font pas systématiquement suivre un « e » à la coupe par une voyelle. Naturellement, la proposition se présente comme un argument d'autorité, qui doit asseoir sa règle en en manifestant l'usage supposé systématique chez les auteurs qui ont des « oreilles doctes $\gg$.

Ces auteurs sont opposés à un autre groupe, celui des « nouveaulx inventeurs », des « nouveaulx controuveurs » ou des « controuveurs de nouveaultez »

4 Il loue ainsi les mérites de Pierre Adam, « marchant et maistre » des Jeux Floraux en 1513 (De Gélis et Anglade 1933, p. 5) ; de Jean Formigail, « maistre » des Jeux floraux en 1513 et 1519 (ibid., p. 5, 13 et 16) ; de Pierre Antoine, maître des requêtes ordinaire du roi, qui est l'un des nombreux hommes de loi, décideurs et/ou participants aux Jeux Floraux de Toulouse. Il évoque aussi Pierre du Faur, docteur ès droits, conseiller au Parlement en 1531, président en 1538, garde des sceaux de la chancellerie de Toulouse en 1539, qui est chancelier des Jeux Floraux de 1535 à 1558 ; Jean de Boyssoné (dit Luscus), conseiller au parlement de Chambéry dès 1539 et mainteneur des Jeux Floraux entre 1520 et 1535 ; ou encore comme Claude de Terlon, avocat au parlement de Toulouse et vainqueur du Souci en 1540 (Guerrier 2003, p. 551 ; Luciani 2006, p. 306-307).

5 Ainsi, chez Jean Meschinot, qui pratique très régulièrement la coupe lyrique (à laquelle Gratien du Pont est défavorable), on trouve aussi la coupe épique, par exemple dans « Je m'esmerveille // comme sur pied me pose » (Meschinot 1493, n. p.).

6 Chez Jean Meschinot, on lit par exemple des vers comme « Par tresgriefve et dure appercevance » ou encore « De luy respondre à ce cas et endroit » (ibid.).

7 Voir par exemple : «Comme maint livre en fait ample rapport», «A tant mys paille et feiz une closture » (Saint-Gelais 1519, n.p.). 
(Du Pont 2012, p. 290, 292 et 294), plus précisément désignés dans les lignes qui suivent où l'auteur incrimine les inventeurs de l'élision à la coupe :

[...] l'origine en est venu de feu maistre Jhan le mere de Belges, Et d'ung Cretin, qui a esté disciple dudict de Belges. Et despuys Clement Marot, lequel confesse en son œuvre de L'adolescence, qu'il ne l'avoit jamais faict que despuys que ledict de Belges luy apprint, et fist faire. (ibid., p. 293)

Les auteurs incriminés ici sont en effet les principaux acteurs de l'abolition de la coupe féminine (Mantovani 2000, p. 73 ; Martinon 1909, p. 65 ; Chamard 1920, p. 171), ce que leur reproche vertement Gratien du Pont.

Si l'on fait le bilan des réponses à la question «cette norme a-t-elle reçu un écho ? », on trouve assez peu de textes contemporains qui correspondent à ce que Gratien du Pont recommande : mis à part le volume des Controverses et la Requeste - qui est une réponse au texte misogyne du Toulousain -, il y a finalement fort peu de choses qui correspondent, en 1539 et même avant, à ce qu'il préconise. Ce constat nous mène à la dernière de nos interrogations : pourquoi cette norme n'a-t-elle pas fonctionné ?

\section{L'ÉCHEC D'UNE ENTREPRISE NORMATIVE}

La question nécessite que l'on s'arrête un instant sur les arguments dont se sert Gratien du Pont pour imposer sa norme, avant d'évaluer les raisons de leur manque d'efficacité.

\subsection{Usage, autorité, noblesse de la langue}

Pour répondre à la question « Qu'est-ce qui a fait que cette norme n'a pas fonctionné ? », je m'inspire ici du passage sur les « vices de impropre locution », qui sont parfois excusables et deviennent alors des figures : « des vices, les ungs s'excusent par figures, et les aultres sont inexcusables, les excusables par figures se font ou bien par coustume que on a d'ainsy faire, ou dire, ou par l'authorité du parlant ou bien par la noblesse et ordonnance d'icelluy langaige, ou qu'il est assez prochain à vertu » (Du Pont 2012, p. 276-277).

Ces critères, empruntés à Quintilien via Pierre Fabri, constituent quatre arguments potentiels pour qu'un énoncé soit considéré comme valide ou ne le soit pas. L'usage (ou la coutume) dont il est question dans le texte de Gratien du Pont semble le fait des poètes anciens, que l'auteur considère comme les seules autorités recevables, seuls capables d'identifier un beau langage, seuls détenteurs de l'indispensable et sélective oreille docte qui sait identifier le langage noble et correctement ordonné, lequel peut être considéré comme " prochain à vertu ». Or, la pratique recommandée ne correspond plus l'usage dominant contemporain, les 
poètes anciens sont loin et une nouvelle langue poétique s'est déjà constituée. $\mathrm{S}$ 'ajoute à cela le fait, régulièrement souligné, que l'auteur est toulousain...

\subsection{Un idéal unifié vs un conflit de langues}

Comme cela est souligné dans son titre, le texte théorique de Gratien du Pont est rédigé en français. Dans les Controverses des sexes masculin et femenin, l'auteur insiste sur ce choix, qui n'est pas celui de sa langue natale (Du Pont 1534, fo ix r ${ }^{\circ}$ ) et qui est à mettre en relation avec les recommandations faites par les organisateurs des Jeux floraux de Toulouse, lesquels préconisent alors l'emploi du français. La langue du Roi progresse dans le sud depuis le $\mathrm{Xv}^{\mathrm{e}}$ siècle. Dans les années 1510, après avoir cohabité quelque temps avec l'occitan, le français supplante quasiment ce dernier dans le concours poétique organisé chaque année à Toulouse (De Gélis 1912, p. 65-68). Le combat pour la langue occitane ne débutera que dans les années 1550, soit onze ans après la parution de l'Art et science de rhetoricque metriffiée, avec l'épître finale de La Requeste faicte et baillée par les dames de la ville de Tolose (1555), dont les choix poétiques peuvent aussi s'expliquer par une revendication identitaire correspondant aux particularismes de la sphère toulousaine partiellement identifiés par Jean Dupré.

Or, comme le constatent Yves Citton et André Wyss pour la période de la Renaissance, il y a « une considérable mystification à parler du français, comme s'il y avait au $\mathrm{XVI}^{\mathrm{e}}$ siècle une norme unique et repérable. La situation est en fait beaucoup plus confuse. Chacun parle - et écrit - le français comme il l'entend, et on ne l'entend pas de la même oreille, suivant qu'on soit né à Paris ou à Lyon » (Citton et Wyss 1989, p. 23). Il y a cependant une norme linguistique qui tend à s'instaurer, laquelle correspond à une unification par l'instauration de la primauté de la langue d'oïl. L'année 1539, qui est celle de la parution de l'Art et science est ainsi (et aussi) celle de la publication de l'ordonnance de Villers-Côterets, qui « prescrit l'emploi exclusif du français dans toutes les pièces judiciaires du Royaume » (Brunot 1906, p. xiii).

Gratien du Pont mène un combat perdu d'avance, qui va à l'encontre de cette unification galopante et des habitudes phonétiques relativement récentes qui s'imposent peu à peu comme la norme, prosodique et poétique. Il en est du reste conscient en soulignant que Fabri, comme les poètes dont il rejette les choix formels, sont « du pays de langue françoyse » :

Lequel Fabri estoit natif du pays de langue françoyse, d'où sont la pluspart de telz nouveaulx inventeurs. (Du Pont 2012, p. 290)

Pour terminer cette réflexion, je reprendrai à mon compte une remarque que fait Gérard Milhe-Poutingon, dans l'introduction au volume Les normes du dire au 
$x v I^{e}$ siècle, dans laquelle il est précisé qu'à la Renaissance, « la langue étant en cours de définition, la grammaticalisation du français repose, dans une large mesure, sur des propositions normatives émanant d'horizons divers et pas toujours conciliables » (Arnould et Milhe-Poutingon 2004, p.8). Les règles que propose Gratien du Pont correspondent ainsi à la tentative d'instaurer une norme, ou plutôt d'entériner une pratique déjà ancienne, régionale, au moment-même où celle-ci est en train de disparaître. Une forme de poésie nouvelle apparaît alors, qui correspond aux habitudes linguistiques et à l'usage de la langue dominante, lesquels diffèrent de ce que Gratien du Pont propose. La variété des recommandations métriques - d'un auteur à l'autre et d'un théoricien à l'autre - dit la disparité des habitudes en matière de prononciation qui coexistent alors. La période du premier $\mathrm{XVI}^{\mathrm{e}}$ siècle est celle d'un travail (théorique et pratique) sur la langue vernaculaire, lequel témoigne d'une volonté manifeste de promouvoir cette dernière - quelle qu'en soit la forme - en résonance avec le pouvoir politique contemporain.

\section{BIBLIOGRAPHIE}

\section{Sources primaires}

Du Pont, Gratien, 2012. Art et science de rhetoricque metriffiée, édition préparée et annotée par Véronique Montagne, Paris, Classiques Garnier.

Du Pont, Gratien, 1534. Controverses des sexes masculin et femenin, Toulouse, Jacques Colomiès.

\section{Sources secondaires}

Arnould, Jean-Claude et Milhe-Poutingon, Gérard (éd.), 2004. Les normes du dire au $X V I^{e}$ siècle, actes du colloque de Rouen (novembre 2001), Paris, Honoré Champion.

Brunot, Ferdinand, 1906. Histoire de la langue française des origines à 1900, t. II, Le seizième siècle, Paris, Colin.

Chamard, Henri, 1920. Les origines de la poésie française de la Renaissance, Lyon, Boccard.

Citton, Yves et Wyss, André, 1989. Les doctrines orthographiques au XVI siècle en France, Genève, Droz.

Cornilliat, François, 2013. «Qu'est-ce qu'un vers pour Jean Bouchet? Autour du Temple de bonne renommée ", L'expérience du vers à la Renaissance, Cahiers V-L. Saulnier $\mathrm{n}^{\circ} 30,57-74$.

Courouau Jean-François (éd.), 2001. Premiers combats pour la langue occitane, Manifestes linguistiques occitans $X V I^{e}-X V I I^{e}$ siècles, Anglet, Atlantica.

Courouau, Jean-François et Gardy, Philippe (éd.), 2003. La Requeste faicte par les dames de la ville de Tolose (1555), Toulouse, Presses universitaires du Mirail.

Dawson, Jean-Charles, 1921. Toulouse in the Renaissance : the floral games, university and students ; Etienne Dolet (1532-1534), New-York, Columbia University press.

Dupré, Jean, 1534. Le palais des nobles dames, Lyon.

Elwert, W. T., 1965. Traité de versification française des origines à nos jours, Paris, Klincksieck.

Gélis, François de, 1912. Histoire critique des jeux floraux depuis leur origine jusqu'à leur transformation en académie (1323-1694), Toulouse, Privat. 
Gélis, François de et Anglade, Jacques (éd.), 1933. Actes et délibérations du collège de rhétorique (1513-1641), Toulouse, Privat.

Fabri, Pierre, 1969. Le grand et vrai art de pleine rhétorique, Genève, Slatkine reprints.

Guerrier, Olivier, 2003. "Autour des Jeux Floraux toulousains : considérations sur l'essai », Arnould Jean-Claude et Mantovani Thierry (éd.), Première poétique française de la Renaissance. Autour des Puys poétiques normands, Actes du Colloque international organisé par le CÉRÉDI (Université de Rouen) octobre 1999, Congrès et conférences sur la Renaissance, $\mathrm{n}^{\circ}$ 33, Paris, Honoré Champion, 543-554.

Langlois Ernest, 1902. L'art et science de rhétorique vulgaire, Recueil d'arts de seconde rhétorique, Paris, Imprimerie Nationale.

Luciani, Isabelle, 2006. « Jeux Floraux et « humanisme civique » au XVI $\mathrm{e}^{\mathrm{e}}$ siècle : entre enjeu de pouvoir et expérience du politique ", L'Humanisme à Toulouse (1480-1596), Actes du colloque international de Toulouse, mai 2004, réunis par Nathalie Dauvois, Paris, Champion, 301-335.

Mantovani, Thierry, 2000. «Pierre Fabri et la poétique des Puys dans le second livre du Grand et vrai art de pleine rhétorique ", Nouvelle revue du XVI siècle, 18/1, 41-54.

Martinon, Philippe, 1909. "La genèse des règles de Jean Lemaire à Malherbe ", Revue d'histoire littéraire de la France, 62-87.

Meschinot, Jean, 1493. Les Lunettes des Princes, Nantes, Larcher.

Milet, Jacques, 1484. L'Istoire de la destruction de Troye la Grant, Paris, Bonhomme.

Monferran, Jean-Charles, 2011. L'École des Muses. Les Arts poétiques français à la Renaissance (1548-1610). Sébillet, Du Bellay, Peletier et les autres, Genève, Droz.

Monferran, Jean-Charles (éd.), 2015. La Muse et le Compas : poétiques à l'aube de l'âge moderne, Paris, Classiques Garnier.

Palsgrave, John, 2003. L'éclaircissement de la langue française (1530), Traduction et notes de Susan Baddeley, Paris, Honoré Champion.

Patterson, W.F., 1935. Three centuries of french poetic theory, a critical history of the chief arts of poetry in France (1328-1630), University of Michigan press.

Rus, Martijn, 1995. De la conception à l'au-delà : textes et documents français d'un siècle, Amsterdam et Atlanta, Rodopi.

Saint Gelais, Octavien de, 1519. Le séjour d'honneur, composé par R. P. en Dieu messire Octovien de Sainct-Gelaiz, évesque d'Angoulesme, Paris, Vérard.

Tervarent, Guy de, 2000. Attributs et symboles dans l'art profane : dictionnaire d'un langage perdu (1450-1600), Genève, Droz. 